\title{
Germanica
}

\section{Les rapports germano-tchèques dans les chroniques d'Ota Filip : une médiation engagée}

The German-Czech relationship in Ota Filip's chronicles: a commitment to Mediation

Das deutsch-tschechische Verhältnis in Ota Filips journalistischen Essays: eine engagierte Vermittlung

Hélène Leclerc

\section{OpenEdition}

Journals

Édition électronique

URL : http://journals.openedition.org/germanica/2006

DOI : 10.4000/germanica.2006

ISSN : 2107-0784

\section{Éditeur}

Université de Lille

Édition imprimée

Date de publication : 31 décembre 2012

Pagination : 135-148

ISBN : 9782913857308

ISSN : 0984-2632

\section{Référence électronique}

Hélène Leclerc, « Les rapports germano-tchèques dans les chroniques d'Ota Filip : une médiation

engagée », Germanica [En ligne], 51 | 2012, mis en ligne le 14 janvier 2013, consulté le 06 octobre 2020 URL : http://journals.openedition.org/germanica/2006 ; DOI : https://doi.org/10.4000/germanica.2006

Ce document a été généré automatiquement le 6 octobre 2020.

(C) Tous droits réservés 


\section{Les rapports germano-tchèques dans les chroniques d'Ota Filip : une médiation engagée}

The German-Czech relationship in Ota Filip's chronicles: a commitment to

\section{Mediation}

Das deutsch-tschechische Verhältnis in Ota Filips journalistischen Essays: eine engagierte Vermittlung

Hélène Leclerc

\section{Ota Filip, « un écrivain de Bohême-Moravie qui vit en Bavière et écrit aussi en allemand $"$.}

Né en 1930 à Ostrava en Moravie d'un père tchèque qui se déclara Allemand en 1939 et d'une mère polonaise $^{2}$, Ota Filip eut pour langue maternelle le tchèque. Son enfance à Ostrava est marquée, comme il le souligne lui-même, par la multiculturalité :

Als ich vor 1939 mit Freunden anderer Nationalitäten und Sprachen Fußball spielte, verständigten wir uns in Ostrava, in einer damals - wie man heute zu sagen pflegt multikulturellen Stadt, auf Tschechisch, Deutsch, Jiddisch und Polnisch. Es war selbstverständlich keine Hochsprache. Ich ,beherrschte‘ zwar damals vier Sprachen, jedoch auf dem Kommunikationsniveau eines neunjährigen Jungen, das alle meine Freunde am linken und am rechten Ufer des Flusses Ostravice sprachen, vor allem die saftigen Schimpfwörter ${ }^{3}$.

Inscrit par son père au lycée allemand le 1er septembre 1939, soit quinze jours avant l'entrée des troupes de la Wehrmacht en Bohême-Moravie, le jeune Ota Filip est brutalement confronté à une langue qu'il ne maitrise pas, mais qu'il finira par apprendre contraint et forcé, expérience traumatique qu'il a notamment relatée dans le « roman autobiographique » Der siebente Lebenslauf publié en 2001.

Après la guerre, Ota Filip fait ses débuts dans le journalisme. Il adhère au Parti communiste tchécoslovaque en 1959, dont il est cependant exclu dès 1960 en raison de 
son attitude critique ; entre 1960 et 1968, il est condamné à deux reprises à des peines de prison et frappé d'interdiction d'écrire. Pendant le Printemps de Prague en 1968, il devient lecteur responsable de la littérature tchèque contemporaine pour la maison d'édition Profil à Ostrava, mais il est arrêté en août 1969 en raison de la publication du roman Blázen ve městě [Un fou dans la ville]. En février 1970, il est de nouveau arrêté et condamné à dix-huit mois de prison; libéré après quinze mois de détention, il doit travailler comme simple ouvrier. En 1974, il émigre en République fédérale d'Allemagne; "dissident malgré lui »", il est alors déchu de la nationalité tchécoslovaque. Installé à Munich où il travaille comme écrivain, journaliste et lecteur dans la maison d'édition S. Fischer, Ota Filip acquiert la nationalité allemande en 1976. Le passage à l'allemand comme langue d'écriture s'effectue progressivement : Ota Filip écrit d'abord des chroniques pour des journaux tels que le Frankfurter Allgemeine Zeitung, puis lorsqu'il constate que celles-ci n'ont plus besoin d'être corrigées par la rédaction, il se lance dans l'écriture de son premier roman en allemand, Großvater und die Kanone, qui paraît en 1981.

Outre l'assurance acquise par le fait que ses chroniques n'étaient plus corrigées par un journal aussi exigeant que le Frankfurter Allgemeine Zeitung, un autre facteur à l'origine de ce changement de langue est qu'Ota Filip n'était plus vraiment satisfait des traductions allemandes de ses romans :

Je länger ich hier bin, desto weniger bin ich mit den deutschen Übersetzungen meiner Romane zufrieden. Diese Unzufriedenheit ging so weit, daß ich mich entschlossen habe, meinen nächsten Roman deutsch zu schreiben ${ }^{5}$.

Le choix de la langue allemande est également à situer dans le contexte de l'émigration qui a suivi les événements de 1968 et ne saurait être interprété comme un fait isolé, comme en témoignent d'autres exemples notoires tels que celui de Libuše Moniková et Jiří Gruša notamment ${ }^{6}$. Un des facteurs déterminants de l'adoption de l'allemand est aussi, comme le soulignent Zbyněk Fišer et Květa Horáčková, l'absence de tout espoir quant à une publication des œuvres de ces auteurs dans l'espace tchécophone ${ }^{7}$.

On peut considérer que l'émigration d'Ota Filip dans la langue allemande a été réussie, si l'on en juge par la reconnaissance littéraire dont il jouit et qui est attestée entre autres par des prix tels que le Prix Chamisso reçu en 1985. Toutefois persiste chez Ota Filip un sentiment d'entre-deux, perçu de plus en plus sinon comme inconfortable, du moins comme troublant, ce qui explique en partie le retour au tchèque opéré par l'auteur depuis quelques années :

In dem Augenblick, als ich dachte, dass ich das Deutsche beherrsche, überkam mich die Angst, dass ich etwas verliere. Ich habe Angst um mein Tschechisch. Schon vor einigen Jahren wurde ich mir bewusst, dass wenn man von einer Sprache in eine andere übergeht, dann entfernen sich einem beide Sprachen irgendwie auf erstaunliche Weise und sind mit einem Mal wie fremd. Jetzt weiß ich nicht, wo ich sprachlich eigentlich zu Hause bin. Aber je älter ich werde, desto mehr neige ich zum Tschechischen ${ }^{8}$.

Par ailleurs, Ota Filip n'a de cesse de souligner les différences irréductibles entre la langue allemande et la langue tchèque :

In den slawischen Sprachen, so auch in der tschechischen, ist es möglich, locker zu schwärmen, man kann vieles schön und poetisch sagen, vor allem das Adjektiv und den Nebensatz aufspielen, ohne dabei auf die Genauigkeit der informellen Aussage viel achten zu müssen. [...] In der deutschen Sprache [...] ist man doch mit der Tradition fest verbunden, mit der Tradition der exakten Denker und des genauen sprachlichen Ausdrucks. Das Deutsche zwang [...] sogar eine andere literarische 
Atmosphäre auf. Diese ist nicht schlechter und nicht besser als die tschechische, sie ist eben anders'.

D'où l'impossibilité pour l'écrivain - en dépit de tentatives renouvelées et d'une volonté de percevoir des racines communes entre les langues ${ }^{10}$ - de traduire lui-même ses romans, qu'il doit alors réécrire, l'illustration de cette impossible auto-traduction étant l'écriture parallèle d'une version tchèque et d'une version allemande $\mathrm{du}$ roman autobiographique Der siebente Lebenslauf / Sedmý Životopis.

Outre ce bilinguisme et cette situation entre deux langues, l'expérience de l'exil est déterminante pour celui qui rejette dorénavant en bloc la notion de patrie, qu'il s'agisse de la patrie politique [Vaterland] ou de celle, plus sentimentale, de Heimat :

[...] ich habe keine Wurzeln nötig. Ich bin ein Mensch, ein zeitgenössischer Nomade zwischen drei europäischen Sprachen und zwei europäischen Kulturen. Ich habe weder eine Heimat, noch ein Vaterland nötig [... ${ }^{11}$.

Le motif du nomade qui apparaît ici rejoint celui du passeur, car ce nomade est paradoxalement bel et bien ancré dans un espace donné, au confluent de deux cultures. Bien qu'Ota Filip ait entre-temps évoqué le trouble dans lequel le plonge désormais sa situation entre deux langues, en 1978, il plaidait ainsi en faveur d'une interculturalité européenne, qui devrait permettre une réconciliation de l'Europe :

$\mathrm{Ob}$ ich tschechisch oder deutsch schreibe, darauf lege ich nicht so viel wert, denn schließlich : Wir alle gehören in das schöne, buntgemischte und aufregende Europa, dessen Traditionen uns verbinden, auch wenn wir in der Vergangenheit so oft versuchten, uns zu trennen, totzuschlagen und zu vernichten. Wir alle haben dafür bezahlt. Soweit meine Kräfte reichen, möchte ich also eine Wiederholung der Irrtümer der Vergangenheit verhindern helfen ${ }^{12}$.

\section{«Une nouvelle vision des rapports tchéco-allemands »} 13 ?

L'Histoire, en particulier l'histoire de Bohême, occupe une place importante dans l'œuvre fictionnelle d'Ota Filip, qu'il s'agisse du personnage de Wallenstein (Valdštýn a Lukrecie [Wallenstein et Lucrèce]), de la fin de l'Autriche-Hongrie et de la Première Guerre mondiale (Großvater und die Kanone), de la Tchécoslovaquie depuis sa fondation (Café Slavia), du Protectorat de Bohême-Moravie et de la Tchécoslovaquie d'après 1945 (Cesta ke hřbitovu [Le chemin qui mène au cimetière], Der Zweikampf, Der siebente Lebenslauf). Doit-on y déceler, comme le formule Jean-Yves Tadié à propos d'auteurs de romans historiques, la volonté d'«explor[er] un passé qui ne passe pas ", "de le regarder en face, ou de le représenter de manière supportable »? Si « à l'origine du genre, à notre époque, il y a toujours une faute originelle ${ }^{14}$, cette faute semble être dans le cas d'Ota Filip la «catastrophe de $1945 »^{15}$ et la tragique fin de la cohabitation séculaire ${ }^{16}$ des Tchèques et des Allemands dans les pays tchèques.

L'histoire de Bohême est en effet interprétée par l'écrivain comme une histoire commune aux Allemands et aux Tchèques. Ainsi rappelle-t-il dans un opuscule consacré à Prague que parmi les rebelles de la révolte des États de Bohême en 1618 se trouvaient des Tchèques et des Allemands, et que les deux nations eurent à subir les conséquences tragiques de la défaite de la Montagne blanche le 8 novembre 1620 :

Über die Exekution der Führer des Aufstandes am Altstädter Ring im Jahr 1621, die

von tschechischer Seite als «Hinrichtung von 27 tschechischen Herren »

bezeichnet wird, bemerkte die Geschichtsschreibung zutreffend: «Insofern die 
Hingerichteten Tschechen waren, waren es keine Herren, also keine Adeligen, und wenn es Herren waren, dann waren es keine Tschechen, sondern Deutsche. » Die Stelle vor dem Rathaus am Altstädter Ring, wo diese 27 «tschechischen Herren" grausam hingerichtet wurden, ist heute mit 27 weißen Kreuzen gekennzeichnet. Es lohnt sich in jedem Fall, die Tafel aus Bronze an der Wand des Rathauses mit den - tschechisierten - Namen der hingerichteten Deutschen zu lesen ${ }^{17}$.

Toutefois, il n'y a nul angélisme sous la plume d'Ota Filip, qui évoque explicitement l'entreprise de germanisation ayant fait suite à la défaite de $1620^{18}$ et précise bien que si Prague était devenue dès le xiIIe siècle une ville bilingue, elle n'en fut jamais pour autant une «ville allemande » ${ }^{19}$. Ota Filip ne succombe pas non plus au «mythe habsbourgeois de la coexistence harmonieuse des nationalités $»^{20}$ puisqu'abordant le xIxe siècle, il écrit :

Ein harmonisches Zusammenleben zwischen Tschechen und Deutschen unter der gütigen Herrschaft der Habsburger gibt es in Prag erst heute, allerdings nur in den Vorstellungen von einigen nostalgisch gestimmten, noch heute «kaisertreuen » Österreichern und Tschechen ${ }^{21}$.

Nous nous concentrons dans cet article sur les chroniques journalistiques d'Ota Filip publiées dans la presse allemande et rassemblées dans les recueils Die stillen Toten unterm Klee. Wiedersehen mit Böhmen ${ }^{22}$ et ... doch die Märchen sprechen deutsch. Geschichten aus Böhmen ${ }^{23}$. Ces chroniques sont particulièrement importantes, ne serait-ce que parce que, comme nous l'avons signalé, Ota Filip en est venu à écrire en allemand précisément via l'écriture de chroniques journalistiques.

Ces deux recueils ont été publiés après la révolution de velours et la chute du régime communiste tchécoslovaque; la plupart des textes qui les constituent, écrits après 1989, évoquent plus de vingt années d'histoire germano-tchèque (le premier recueil concerne une période s'étendant de décembre 1979 à décembre 1991, le second, d'avril 1976 à juillet 1996). Les recueils présentent un classement de textes a priori arbitraire car ni chronologique, ni thématique ; la datation précise en a été effacée, de même que la provenance originelle des textes ${ }^{24}$, comme par volonté de composer une nouvelle œuvre, une œuvre en soi. Les recueils empruntent chacun leur titre à l'une des chroniques. Le premier, Die stillen Toten unterm Klee, désigne l'endroit où ont été enterrés les Allemands de Brno (Brünn) morts d'épuisement lors de leur marche forcée le 30 mai 1945. À cette masse anonyme et silencieuse, reposant désormais pour toujours dans la terre de Moravie, paraît répondre le titre du second recueil ... doch die Märchen sprechen deutsch : ces morts ont certes été privés de sépulture véritable, de leur identité et de parole, ils gisent muets "sous un champ de trèfle ", mais les contes continuent à résonner en allemand.

En dépit d'une composition à première vue aléatoire, le fil conducteur des deux recueils apparaît clairement ${ }^{25}$. Ota Filip y aborde en effet les relations germano-tchèques/ tchécoslovaques depuis 1945 et surtout depuis 1989, les expulsions des Allemands des Sudètes et leurs conséquences. L'ouverture des frontières en 1989 a donné lieu à la réaffirmation de revendications de la part des associations d'Allemands des Sudètes et à la résurgence des peurs tchèques, engendrant crispations de part et d'autre ${ }^{26}$. Le contentieux n'a pas été levé par la signature du traité de bon voisinage entre l'Allemagne et la Tchécoslovaquie en 1992, ni complètement non plus par celle de la «déclaration de réconciliation germano-tchèque » en 1997, pour laquelle Ota Filip militait et à laquelle il fait allusion dans la dernière chronique du second recueil ${ }^{27}$. Le 
lien thématique ténu entre les deux recueils ressort par ailleurs de la reprise dans le second, en exergue du livre, du titre de la préface du premier: "L'hostilité entre Tchèques et Allemands doit être enterrée » [Zwischen Tschechen und Deutschen muß die Feindseligkeit begraben werden]; le message et l'ambition sont d'emblée affichés dans les deux cas, le modal « muß » signale qu'il s'agit bien d'une œuvre militante.

L'intention de l'auteur est alors double : d'une part, lutter contre l'oubli - tel est bien le leitmotiv -, oubli des crimes passés et oubli de cette culture allemande de Bohême dont ne subsistent que des vestiges, et, d'autre part, parvenir au pardon et à la possibilité de rapports harmonieux entre individus, jeter les bases de la réconciliation. Le titre de la préface du premier recueil est en cela programmatique, de même que les dernières lignes de la préface du second:

Immer wieder habe ich in den Ruinen, die uns die tschechisch-deutsche Vergangenheit in meiner einstigen Heimat nach 1939 hinterlassen hat, Gemeinsamkeiten entdeckt, verschüttete Grundmauern, abendländisch-christliche Traditionen, auf welchen wir eine gemeinsame Zukunft in Europa aufbauen können. (Filip 1996 : 12)

De cette volonté de vaincre l'oubli participe la démarche consistant à fixer en recueil des chroniques relevant par nature de l'éphémère ${ }^{28}$. Justifiant et légitimant cette démarche, J. G. Reißmüller note, rapportant les propos d'Ota Filip :

Wer heute in Böhmen und Mähren unter Fünfzig ist, der weiß in der Regel nichts vom Unrecht an den böhmischen und mährischen Deutschen, und viele wollen auch nichts davon wissen. [...] Viele Prager glauben, Deutsche seien in ihre Stadt erst unter Hitler gekommen. In den Schulbüchern der Tschechoslowakei finde sich kein Wort über den Beitrag der Deutschen zur Geschichte des Landes. Daran wird sich nichts ändern, fürchtet Filip, solange die Politiker in der Tschechoslowakei, außer einigen wenigen, die Sache mit den Deutschen umgehen. (Filip 1992 : 11-12)

Cette ambition médiatrice et conciliatrice, qui passe obligatoirement par une nouvelle façon d'aborder la question des expulsions, n'est cependant pas propre à Ota Filip : la «nouvelle vision» des relations germano-tchèques est à porter au crédit de la dissidence tchécoslovaque ${ }^{29}$ et de l'un de ses plus illustres représentants, Václav Havel, dont l'action en ce sens est du reste maintes fois rappelée dans les chroniques. Ota Filip s'inscrirait ainsi dans une lignée, comme le souligne d'emblée J. G. Reißmüller :

Die Prager Dissidenten und das tschechische Exil haben in ihrer Mehrheit die Vertreibung der Deutschen aus der Tschechoslowakei nach dem Zweiten Weltkrieg als Unrecht, mindestens als politischen Fehler erkannt. Filip weiß es; beiden Gruppen hat er nacheinander angehört. (Filip 1992 : 9)

Les conséquences des expulsions ont été dramatiques, bien sûr d'abord pour les expulsés, mais aussi - et c'est ce que martèle Ota Filip dans les deux recueils - pour les territoires laissés à l'abandon, voire pour les Tchèques eux-mêmes. C'est ainsi que l'écrivain chroniqueur dresse une liste qui semble infinie, d'églises, couvents et châteaux en ruine ${ }^{30}$, mais la ruine est aussi écologique ${ }^{31}$ et morale. Ota Filip décrit en effet la force du ressentiment tchèque qui empêcherait selon lui d'aller de l'avant :

Von den achthundert Jahren Tradition blieben innerhalb der historischen Grenzen des einstigen böhmischen Königreichs nach 1945 nur ein geschichtsträchtiger Trümmerhaufen, eine leidenschaftliche Feindschaft und ein tiefes Mißtrauen zurück. (Filip $1992: 67$ )

Ce motif de la méfiance tchèque à l'égard des Allemands apparaît de nouveau dans le second recueil à propos du maire de Děč́n qui souhaiterait rappeler le passé de sa ville ; 
un prêtre avec lequel s'entretient Ota Filip juge que seul un miracle pourrait combler le fossé qui sépare Allemands et Tchèques :

Christliche Liebe ist zwar die Voraussetzung für Versöhnung. Aber Liebe allein reicht zur Überwindung des Grabens zwischen Deutschen und Tschechen nicht aus.

(Filip 1996 : 131)

L'impression qui domine à la lecture de ces chroniques est celle d'une désolation complète, d'un désastre humain, culturel et écologique. Toutefois, Ota Filip parvient à montrer, par petites touches, les avancées - certes encore bien fragiles à l'époque où il écrit - d'une réconciliation germano-tchèque. En rappelant l'engagement de la dissidence tchécoslovaque et sa prise de distance vis-à-vis des expulsions, et en reprenant pour ce faire des chroniques plus anciennes (de décembre 1979, octobre 1980 et avril 1983) ${ }^{32}$, Ota Filip révèle malgré tout l'ancrage de l'ambition de réconciliation dans la société tchèque. En évoquant des initiatives telles que la fondation de l'Adalbert-Stifter-Verein à Český Krumlov (Krumau) et l'organisation dans cette même ville ainsi qu'à Linz de deux expositions bilingues, l'une consacrée à Stifter, l'autre à Egon Schiele (Filip 1992: 40-45), en décrivant les entreprises de restauration de monuments en ruine (Filip 1992: 74-84) et de coopération germano-tchèque qui parviennent à se mettre en place comme à Tachov (Tachau) (Filip 1992:65-73) ou les avancées du travail de mémoire, comme à Ustí nad Labem (Aussig) avec l'apposition d'une plaque en allemand commémorant le massacre d'Allemands le 31 juillet $1945^{33}$, en mettant en avant des Tchèques disposés à la réconciliation ${ }^{34}$, en rapportant l'histoire de ce couple d'Allemands des Sudètes retourné à Schönwald (Lesná) où il a été chaleureusement accueilli par les villageois tchèques (Filip 1996: 75-81), ou encore en consacrant plusieurs chroniques à des figures symbolisant la médiation ${ }^{35}$, Ota Filip s'attache à débusquer les raisons d'espérer. Et ce n'est sans doute pas un hasard qu'il ait choisi de clore le recueil Die stillen Toten par une chronique dont la dernière phrase contient le terme même d'espoir :

[...] schon die Tatsache, daß Tschechen von Rang und Namen [...] offiziell im Auftrag der Regierung mit den Deutschen und mit der verschwiegenen deutschen Minderheit offen über ihre Auferstehung und über ihre Zukunft in der erneuerten tschechisch-slowakischen Demokratie sprachen, war nach vier Jahrzehnten des Schweigens, des Verschweigens und Totschweigens eine hoffnungsvolle Antwort. (Filip 1992 : 224)

De la même façon, le second recueil s'achève par une chronique plus positive, qui contraste fortement avec la plupart des précédentes. Cette chronique est consacrée à l'acte de renonciation solennelle, effectué en juin 1996 par la famille Thun-Hohenstein, à toute indemnisation ou restitution des biens confisqués lors de son expulsion en 1945. Ota Filip y rend un hommage appuyé à cette famille et, à travers elle, à la tradition bohémiste ${ }^{36}$ et à ses potentialités conciliatrices; il rappelle en effet l'engagement patriotique bohème des ancêtres du prince Thun-Hohenstein, notamment celui de Franz Thun ${ }^{37}$ durant le Vormärz, dans la tradition duquel le Dr. Thomas ThunHohenstein se situe explicitement :

Unser Verzicht ist heute ein Versuch, einen wichtigen Teil unserer Familientradition [...] für Ausgleich und Vermittlung zwischen Deutschen und Tschechen einzusetzen. (Filip $1996: 207$ )

Dans cette dernière chronique, Ota Filip se tient en retrait et restitue les propos du Dr. Thomas Thun-Hohenstein; c'est l'appel à la réconciliation lancé par ce dernier qui clôt la chronique. C'est du reste la position généralement adoptée dans la plupart des chroniques par le médiateur que l'écrivain aspire à être. Ota Filip n'a de cesse de 
donner la parole aux personnes qu'il rencontre, qu'elles soient tchèques ou allemandes, de restituer leurs propos par le biais du discours direct; nombre de chroniques fonctionnent sur le mode de l'interview, et le chroniqueur s'abstient de commenter les propos qu'il recueille. Bien sûr, il serait naiff de conclure à la neutralité absolue de l'auteur qui se contenterait d'un rôle de porte-voix ; il y a bel et bien prise de position de la part d'Ota Filip, mais celle-ci se fait de manière discrète et réside dans la sélection des informations rapportées et dans la structure des chroniques ainsi que du recueil. Comme l'analyse $\mathrm{Ph}$. Blondeau, le genre de la chronique se caractérise en effet par « une subjectivité affirmée, puisqu'il n'y a pas de chronique sans la présence forte d'un auteur qui, en un sens, la légitime $»^{38}$. Dans les chroniques d'Ota Filip, l'usage de la première personne est cependant très mesuré ; l'apparition du «je » n'en est dès lors que plus remarquable. Aussi est-il intéressant de constater que le recueil Die stillen Toten unterm Klee s'ouvre sur un souvenir d'enfance et sur l'emploi, dès l'incipit, du pronom « je $»$ :

Vor dem Krieg, ich bin damals acht gewesen, nahm mich meine Mutter auf ihre, wie sie sagte, Bildungsfahrt in drei Kirchen in Westböhmen mit. (Filip 1992 : 15)

Néanmoins, c'est pour mieux disparaître ensuite et ne resurgir vraiment que dans la onzième chronique qui, de nouveau, évoque un souvenir personnel de l'auteur, puis dans la dix-neuvième chronique, sans doute la plus importante car il s'agit non seulement de la plus longue, mais surtout de celle qui confère son titre au recueil. Pourquoi ouvrir le recueil par un texte qui n'est ni le plus ancien ni le plus récent du livre, ni la chronique-titre, sinon pour, peut-être, placer l'ensemble du recueil sous le signe d'une subjectivité assumée? Ce premier texte est en effet structuré par un contraste appuyé entre les souvenirs d'enfance lumineux de l'auteur - la visite de l'église de Horšovský Týn avec sa mère en 1938 -, scandés par l'expression «ich erinnere mich », et l'état présent, désolé du lieu :

Ich erinnere mich: Kirchweih in Lestkov vor zweiundfünfzig Jahren : Ich sitze mit Mutter unter einem grünweißen Sonnenschirm. Die Kirche strahlt in der Sonne mit ihrem warmen Gelb. [...] Jetzt ist das mit Schindeln gedeckte Dach der Kirche verfault und droht einzustürzen. (Filip $1992: 19)$

Par ce « je », d'emblée présent, l'auteur s'engage. En tentant de se placer au-dessus des deux camps, il opte toutefois pour une position paradoxale que Massum Faryar a, à juste titre, identifiée comme celle d'un "narrateur omniscient à la première personne " ${ }^{39}$. Il se place aussi dans une position dialogique vis-à-vis des lecteurs, et la question du destinataire se pose. A priori, le lectorat est allemand, puisque les chroniques sont initialement parues dans la presse allemande; par ailleurs, contrairement aux romans d'Ota Filip, ces recueils n'ont pas été traduits en tchèque. L'entreprise de médiation ne serait-elle pas, alors, d'emblée biaisée ?

D'aucuns ont pu considérer qu'Ota Filip faisait preuve de sévérité à l'égard des Tchèques. J. G. Reißmüller indique qu'Ota Filip se demande si « les Tchèques n'ont pas tout simplement perdu au cours du dernier demi-siècle tout sens de la morale et de la justice» (Filip 1992 : 10), et le critique Christophe Bartmann a formulé le jugement suivant :

Das erklärt vielleicht, weshalb er mit den Tschechen so streng ins Gericht geht und den Deutschen gegenüber Milde walten läßt. Den Deutschen hilft es beim Abbau von schlechtem Gewissen gegenüber den Tschechen. Den Tschechen hingegen soll es zur Warnung gereichen. [...] Aber das Buch gibt zuwenig Auskunft über ganz normale Menschen, die tschechisch sprechen und nicht vom volkstümlichen 
Deutschenhaß befallen sind. Fast möchte man Filip um Gerechtigkeit für die Tschechen bitten ${ }^{40}$.

Renata Cornejo est aussi d'avis que l'image des Allemands véhiculée par Ota Filip serait plus favorable que celle des Tchèques, même si, précise-t-elle, Ota Filip se garde bien de parler des Allemands ou des Tchèques de manière globale et évoque au contraire toujours des destins individuels ${ }^{41}$. En réalité, bien plus qu'aux Tchèques, l'écrivain s'en prend aux " passéistes » [Ewiggestrige] tchèques, qui refusent catégoriquement toute perspective de réconciliation avec l'Allemagne, comme il le soulignait encore au moment de la signature de la déclaration de réconciliation germano-tchèque :

Als ich in den vergangenen Wochen und noch am Tag der Unterzeichnung der deutsch-tschechischen Erklärung das aggressive Gerede der tschechischen, marxistisch-faschistoid-sozialdemokratischen Front hörte und las, gewann ich den Eindruck, daß sich das Häuflein aus pensionierten tschechischen Kommunisten, Prager faschistoiden Republikanern und dem politisch rückständigsten Teil der tschechischen Sozialdemokratie unbedingt immer noch im Kriegszustand mit den Sudetendeutschen sehen will. Der Zweite Weltkrieg ist für diese Ewiggestrigen noch nicht zu Ende. Sie möchten, koste es, was es wolle, in der allerletzten Schlacht gegen die Sudetendeutschen triumphieren, auch wenn dabei schon die erreichte Aussöhnung zwischen Sudetendeutschen und Tschechen, nebenbei auch die deutsch-tschechische Erklärung, in Scherben gehen sollte ${ }^{42}$.

Dans l'ambition médiatrice d'Ota Filip, la chronique journalistique prend le relais de la littérature, la médiation devient directe et, partant, bel et bien engagée ; le médiateur dénonce avec conviction et parfois virulence des comportements qu'il juge dangereux, au risque de s'exposer lui-même à la critique et de susciter des inimitiés ${ }^{43}$.

\section{NOTES}

1. «Ich bin jetzt wieder ein böhmisch-mährischer Schriftsteller, der in Bayern lebt und auch Deutsch schreibt ", interview accordée à Renata Cornejo le 4 septembre 2008 dans l'appartement de l'auteur à Murnau (Bavière) et publiée dans: Renata Cornejo, Heimat im Wort. Zum Sprachwechsel der deutsch schreibenden tschechischen Autorinnen und Autoren nach 1968. Eine Bestandsaufnahme, Wien, Praesens, 2010, pp. 420-443 (ici p. 443). Le qualificatif «böhmischmährisch » employé ici par Ota Filip est intéressant car ce dernier est né dans la partie silésienne de l'ex-Tchécoslovaquie et, ailleurs, il se présente volontiers comme un écrivain tchèque [tschechisch] (ex. p. 439 de la même interview). Par ce terme, Ota Filip semble vouloir éviter l'usage de catégories nationales et préférer penser en termes d'espace ; cela rejoint son rejet des notions de "Vaterland» et de "Heimat» (cf. infra). Pour une présentation biographique d'Ota Filip, voir notamment James Jordan, «Ota Filip», in Kritisches Lexikon zur deutschsprachigen Gegenwartsliteratur, 10/98, S. 2-11.

2. Voir l'interview accordée à Renata Cornejo (cf. note 1), p. 425

3. Ibidem, p. 426.

4. Expression récurrente chez Ota Filip, qu'il applique tantôt à son propre cas (Ota Filip, "Dissident wider willen», in Karl Corino (éd.), Autoren im Exil, Frankfurt am Main, Fischer Taschenbuch Verlag, 1981, pp. 37-45), tantôt à des écrivains tchécoslovaques non exilés (Ota Filip, Pavel Tigrid, «Dissidenten wider willen», préface du recueil Kontinent. Sonderband Prag. 
Unabhängiges Forum nicht-exilierter tschechoslowakischer Autoren, Frankfurt am Main, Ullstein, 1976, pp. 7-15).

5. Ota Filip, « Dissident wider willen », in Karl Corino (éd.), op. cit., p. 42.

6. Voir Alfrun Kliems, Im Stummland. Zum Exilwerk von Libuše Moniková, Jiř́ Gruša und Ota Filip, Frankfurt/Main, Peter Lang, 2002, et Renata Cornejo, Heimat im Wort. Zum Sprachwechsel der deutsch schreibenden tschechischen Autorinnen und Autoren nach 1968. Eine Bestandsaufnahme, op. cit.

7. Zbyněk Fišer, Květa Horáčková, "Tschechische Literatur in deutscher Sprache ", in Walter Koschmal, Marek Nekula et Joachim Rogall (éds.), Deutsche und Tschechen. Geschichte, Kultur, Politik, München, Beck, 2003, p. 290-301 (ici p. 291).

8. Cf. interview note 1, p. 440.

9. Ota Filip, Großvater und die Kanone, 1981, Frankfurt am Main, Fischer Taschenbuch Verlag, 1983, p. 155.

10. Ota Filip écrit ainsi : «Ich gehe von dem Standpunkt aus, daß wir Westeuropäer aus einem Nest kommen. Die deutsche Sprache empfinde ich nicht als fremd, sie klingt nur anders als die tschechische, ihre geistigen Wurzeln weisen Gemeinsamkeiten auf, die nicht zu übersehen sind. ", in Karl Corino (éd.), op. cit., p. 42.

11. Ota Filip, « $O$ du mein liebes fremdsprachiges Land », in Volker Heigenmooser et Johann P. Tammen (éds.), Verlegen im Exil. Reden, Vorträge, Statements, Fakten und Fiktionen, Lyrik und Prosa. Dokumentation des Bremerhavener P.E.N.-Symposiums 1997, Bremerhaven 1997. Cité par Alfrun Kliems, op. cit., p. 209.

12. Ota Filip, « Dissident wider willen », in Karl Corino (éd.), op. cit., p. 45.

13. C'est ce qu'aurait proposé Ota Filip dans le roman Cesta ke hřbitovu d'après Hana VoisineJechová, Histoire de la littérature tchèque, Paris, Fayard, 2001, p. 667.

14. Jean-Yves Tadié, «Les écrivains et le roman historique au $\mathrm{xx}^{\mathrm{e}}$ siècle. Esthétique et psychologie », in Le Débat, n¹65 («L'histoire saisie par la fiction »), mai-août 2011, pp. 136-145 (ici p. 144).

15. Sur ce terme de "catastrophe", voir notamment: Gemeinsame deutsch-tschechische Historikerkommission (éd.), Konfliktgemeinschaft, Katastrophe, Entspannung. Skizze einer Darstellung der deutsch-tschechischen Geschichte seit dem 19. Jahrhundert, München, Oldenburg, 1996.

16. Les Allemands se sont installés en Bohême au XIII siècle à l'invitation des souverains přemyslides tchèques.

17. Ota Filip, Mein Prag. Fotografiert von Michael Schilhansl, Dortmund, Harenberg Edition, 1992, p. 15.

18. Ibidem, p. 16.

19. Ibidem, p. 17.

20. Sur ce mythe décrit par Claudio Magris, voir notamment Jacques Le Rider, «Le mythe habsbourgeois de la coexistence harmonieuse des nationalités et ses principes antagonistes : le colonialisme et le nationalisme », in Herta Luise Ott, Marc Beghin (dir.), Penser le pluriculturel en Europe centrale, in Chroniques allemandes n¹1, 2006-2007, pp. 73-84.

21. Ota Filip, Mein Prag, p. 19.

22. Ota Filip, Die stillen Toten unterm Klee, München, Langen Müller, 1992. Dorénavant cité ainsi : Filip 1992

23. Ota Filip, ... doch die Märchen sprechen deutsch. Geschichten aus Böhmen, München, Langen Müller, 1996. Dorénavant cité ainsi : Filip 1996.

24. « in bundesdeutschen Zeitungen, Zeitschriften und im Rundfunk» indique simplement Ota Filip sur la jaquette du recueil Die stillen Toten unterm Klee. La jaquette du second précise FAZ, Die Zeit, Die Welt.

25. «Le recueil qui rassemble a posteriori les chroniques, conférant de la durée à l'éphémère, induit [...] un type particulier de lecture : une lecture fragmentée, discontinue, et pourtant unie, analogue à celle d'un ensemble de poèmes ou de nouvelles composant un même ouvrage », in 
Bruno Curatolo, Alain Schaffner, «La chronique journalistique, un (mauvais) genre littéraire? », in Bruno Curatolo, Alain Schaffner (dirs.), La Chronique journalistique des écrivains (1880-2000), Éd. universitaires de Dijon, 2010, p. 8.

26. Sur ces questions, voir les travaux d'Anne Bazin, en particulier «Tchèques et Allemands aujourd'hui : bon voisinage sur fond de réconciliation difficile ", in : Revues d'Études comparatives Est-Ouest, vol. 40, n¹, mars 2009, p. 99-120.

27. Cf. Hélène Leclerc, «La "déclaration de réconciliation" germano-tchèque du 21 janvier 1997, un tournant dans les relations germano-tchèques?", in Revue d'Allemagne et des pays de langue allemande, tome 43, 2-2011, p. 265-275.

28. «Par définition, la chronique journalistique relève d'abord de l'éphémère, et le chroniqueur n'existe véritablement que lorsque sa parole acquiert une stabilité en se fixant en recueil. Il ne paraît pas que tous les chroniqueurs aient eu l'ambition de se faire reconnaître cette facette de leur talent par la postérité ». In Alain Cresciucci, «La chronique comme œuvre. Pour saluer Philippe Muray (1945-2006)», in Curatolo, Schaffner, op. cit., p.111-119 (ici p. 112). Plus que l'ambition de faire reconnaître ces chroniques par la postérité, ce qui semble présider à la publication sous forme de recueil, c'est la volonté d'Ota Filip de continuer à alerter sur la nécessité d'une réconciliation entre Tchèques et Allemands.

29. Cf. Anne Bazin, «Tchèques et Allemands sur la voie d'une difficile réconciliation », in Relations internationales et stratégiques, $n^{\circ} 26,1997$, p. 154-163.

30. Le titre même des chroniques met souvent l'accent sur ce motif de la ruine : «Kleinseitner Geschichten - Verfall hinter Fassaden », «Eine Ruine in Tachau - Die Reithalle der Fürsten zu Windischgrätz » (Filip 1992: 99-108; 116-119); «Zur Kaserne degradiert, ausgeraubt und zur Ruine verkommen - Das Kloster Tepl», «Ein Ruinendorf im Königswald - Prosau bei Marienbad ", « ... doch die Märchen sprechen deutsch - Auf den Spuren einer verschwundenen Kultur » (Filip 1996 : 36-44, 56-58, 107-123).

31. Par exemple dans la chronique « Nach Kleinaupa kommt keiner zurück. Ein kleines Dorf im Riesengebirge ", (Filip 1992: 196-202). Dans ce village, le désastre écologique est tel que les Tchèques n'ont pas à craindre que les Allemands y reviennent. À Aussig (Ustí nad Labem), les habitants seraient, au moment où la chronique est écrite, en juillet 1991, plus préoccupés par le désastre écologique auquel ils sont confrontés que par le passé (Filip 1992 : 127).

32. « Nachdenken über ein Tabu. Tschechische Oppositionnelle greifen das Thema Vertreibung auf»; «Gespräche und Streitgespräche. Die unbewältigte böhmische Vergangenheit », «Danubius. Gewissen eines Volkes » et «Schlechtes Gewissen, schmutzige Hände. Ein Gespräch mit Jan Mlynarik» (Filip 1992 : 34-39, 46-58, 203-208, 209-213).

33. " Erschlagen und in die Elbe geworfen. Dem Massenmord von Aussig auf die Spur» (Filip 1992 : 120-127). Dans cette chronique, Ota Filip confronte plusieurs témoignages et plusieurs travaux d'historiens, tchèques et allemands. Sur les légendes entourant ce massacre, voir Eva et Hans Henning, Die Vertreibung im deutschen Erinnern. Legenden, Mythos, Geschichte, Paderborn, F. Schöningh, 2010.

34. Tel le poète Petr Pavlík, qui déclare : «Wenn wir uns an nichts, was die Deutschen in Böhmen aufgebaut haben, erinnern dürfen, dann müssten wir halb Prag und achthundert Jahre aus der böhmischen Geschichte vergessen ! » (Filip $1992: 81$ ).

35. Le traducteur F. P. Künzel (Filip 1996: 13-22), le dernier poète allemand de Prague Jiř́ Havliček (Filip 1996 : 124-129), voire Saint Jean Népomucène (Filip 1996: 150), ou encore les nombreuses figures de religieux rencontrés par le chroniqueur.

36. Le bohémisme renvoie à une situation antérieure à l'émergence des nationalismes allemand et tchèque et des conflits nationaux qui se cristallisent à partir de 1848 . Le bohémisme se caractérisait par la défense d'un patriotisme commun, d'une culture et de valeurs communes aux Allemands et aux Tchèques en Bohême. Cf. Hélène Leclerc, Une littérature entre deux peuples. Écrivains de langue allemande en Bohême 1815-1848, Toulouse, Presses Universitaires du Mirail, 2011. 
37. Le frère du comte Franz Thun, Mathias, est l'auteur de la célèbre formule bohémiste : « Ni Tchèque, ni Allemand, mais Bohème ». « $[\mathrm{Er}]$ versuchte [...] im eskalierten Nationalitätenkonflikt, zwischen Tschechen und Deutschen zu vermitteln » (Filip 1996 : 204).

38. Philippe Blondeau, «Avatars de la chronique chez Pierre Mac Orlan », in Curatolo, Schaffner, op. cit., p. 123-133 (ici p. 123).

39. Massum Faryar, Fenster zur Zeitgeschichte: eine monographische Studie zu Ota Filip und seinem Werk, Berlin, Mensch-und-Buch-Verlag, 2005, p. 272.

40. Christophe Bartmann, «Die Antwort weiß der Koksofen. Versöhnung ist schwierig: Ota Filip erzählt Geschichten aus Böhmen », in Frankfurter Allgemeine Zeitung, 12 décembre 1996, n²90, p. 36.

41. Cf. R. Cornejo, op. cit., p. 316.

42. Ota Filip, «Wenn man “odsun” und "vyhnání” kreuzt, kommt "vyhánení" heraus. Der Streit um Abschiebung und Vertreibung, die philologischen Finessen und ihre glorreiche Auflösung in der deutsch-tschechischen Erklärung", in Frankfurter Allgemeine Zeitung, 24 décembre 1996, $\mathrm{n}^{\circ} 300, \mathrm{p} .4$.

43. La polémique dont Ota Filip a fait l'objet en 1998 à la suite du documentaire Der lachende Barbar de Martin Möller, l'accusant d'avoir collaboré avec les services secrets tchécoslovaques dans les années 50 puis 70 - accusations graves qui ont provoqué le suicide du fils d'Ota Filip et auxquelles il a réagi par l'intermédiaire du roman Der siebente Lebenslauf - constituerait selon l'écrivain « un acte de vengeance après-coup, orchestré par ceux qui étaient au pouvoir jusqu'en 1989 et qui aujourd'hui encore cherchent à manipuler »; Ota Filip ajoute : "J'ai été trop actif à leurs yeux et j'ai donné mon avis sur la relation germano-tchèque et les Allemands des Sudètes. Cela a déplu à beaucoup. À présent, ils voient une occasion de me détruire. » In « Man widersteht dem Druck nicht », interview pour l'hebdomadaire allemand Der Spiegel, 3/1998, p. 163.

\section{RÉSUMÉS}

L'écrivain d'origine tchèque Ota Filip, né en 1930, émigré en République fédérale en 1974 et naturalisé en 1976, est l'auteur d'une œuvre littéraire écrite tour à tour en tchèque et en allemand. C'est par l'écriture de chroniques journalistiques dans la presse allemande qu'il s'est progressivement tourné vers l'allemand comme langue d'écriture. Ces chroniques, pour la plupart consacrées aux relations germano-tchèques depuis 1945, ont été publiées sous la forme de deux recueils en 1992 et 1996, dans le contexte d'une redéfinition de ces relations à la suite de l'effondrement des régimes communistes. Après avoir présenté les conditions propices à une démarche médiatrice qui caractérisent l'écrivain Ota Filip (situation de multiculturalité, exil, changement de langue), l'article examine comment cette ambition de médiation se met en œuvre dans ces deux recueils et quelles spécificités le genre de la chronique permet. Ota Filip plaide avec vigueur et non sans sévérité pour une réconciliation entre Allemands et Tchèques, le tableau de la ruine morale qui est dressé laissant malgré tout entrevoir des motifs d'espoir.

Ota Filip, who was born in Czechoslovakia in 1930, immigrated to Western Germany in 1974 and was granted the German citizenship in 1976. His fiction is written in Czech as well as in German. He progressively turned to writing his novels in German after writing chronicles for German newspapers. These chronicles, which are mainly dedicated to the German-Czech relationship since 1945, were published as books in 1992 and 1996, in the context of a redefinition of this 
relationship after the fall of European socialist regimes. After presenting the conditions that give Ota Filip the possibility of playing the role of a mediator (multiculturality, exile, change of language), the article examines the mediation process in these two books, emphasizing the particularities of the genre of the chronicle. In both books Ota Filip vigorously calls for reconciliation between Germans and Czechs, denouncing moral ruin but showing at the same time there are reasons for hope.

Dem Schriftsteller tschechischer Herkunft Ota Filip (1930 geboren), der 1974 in die Bundesrepublik auswanderte und 1976 die deutsche Staatsbürgerschaft erhielt, ist ein auf Tschechisch und Deutsch verfasstes literarisches Werk zu verdanken. Durch die Publizierung von Essays in verschiedenen westdeutschen Zeitungen ermutigt, fing er allmählich an, auch Romane auf Deutsch zu schreiben. Diese Pressebeiträge, die überwiegend den deutsch-tschechischen Beziehungen seit 1945 gewidmet sind, wurden 1992 und 1996 im Kontext einer Neugestaltung dieser Beziehungen nach dem Zusammenbruch der kommunistischen Regimes als Sammelbände erneut veröffentlicht. Nachdem verschiedene eine Vermittlung ermöglichende Faktoren wie Multikulturalität, Exil und Sprachwechsel präsentiert werden, wird der Vermittlungsprozess in den beiden Bänden untersucht, wobei auf die Spezifika der Gattung besonders hingewiesen wird. In beiden Bänden plädiert Filip vehement und streng für eine Aussöhnung zwischen Deutschen und Tschechen, wobei das von ihm entworfene Bild der moralischen Ruine trotz alledem durch einiges optimistisches Licht beleuchtet wird.

\section{AUTEURS}

\section{HÉLÈNE LECLERC}

Université de Toulouse 2 